\title{
Effect of Probiotics (Saccharomyces cerevisiae) Supplementation on Growth Performance of Growing Barbari and Sirohi Goats
}

\author{
V. N. Gautam*, Shraddha Shrivastava, G. P. Lakhani, R. P. S. Baghel, B. Roy, \\ A. Jain and Rahul Shehar \\ Department of L.P.M., College of Veterinary Science and Animal Husbandry, \\ N.D.V.S.U., Jabalpur, India \\ *Corresponding author
}

\section{A B S T R A C T}

\section{Keywords}

Probiotic supplements, Weight gain, Body weight gain, Body length, Sirohi

Article Info

Accepted: 04 November 2019 Available Online: 10 December 2019
The study was conducted to evaluate the influence of probiotic supplementation on body weight, body weight gain, body length, Sirohi and Barbari kids just after weaning at the age of 2-3 month of either sex were selected and distributed randomly i.e. Twenty four (24) Sirohi and Twenty four (24) Barbari male and female kids were randomly selected from the Institute's flock for the study. Average body weight $(\mathrm{kg})$ of Barbari kids at the end of experiment was $14.38 \pm 0.24,15.25 \pm 0.23,15.81 \pm 0.26,16.15 \pm 0.19$ and Sirohi kids was $15.00 \pm 0.24,15.87 \pm 0.23,16.42 \pm 0.26,16.77 \pm 0.91$ in $\mathrm{T}_{\mathrm{i}}, \mathrm{T}_{2}, \mathrm{~T}_{3}$ and $\mathrm{T}_{4}$ groups respectively. The overall body weight gain $(\mathrm{kg})$ during experimental period of Barbari kids was $0.55 \pm 0.01,0.72 \pm 0.03,0.76 \pm 0.03$ and $0.82 \pm 0.03 \mathrm{~kg}$ and of Barbari kids was $0.55 \pm 0.01,0.55 \pm 0.02,0.76 \pm 0.03$ and $0.82 \pm 0.03$ for $\mathrm{T}_{1}, \mathrm{~T}_{2}, \mathrm{~T}_{3}$ and $\mathrm{T}_{4}$ group respectively. At the end of experiment average body length $(\mathrm{cm})$ in Barbari kids were recorded as $57.58 \pm 0.25,60.20 \pm 0.53,60.26$ \pm 0.31 and $59.40 \pm 0.59$ and in Sirohi kids were recorded as $59.04 \pm 0.25,61.66$ $\pm 0.53,61.66 \pm 0.53$ and $60.86 \pm 0.59$ for $T_{1}, T_{2}, T_{3}$ and $T_{4}$ group of respectively.

\section{Introduction}

Probiotics are non-pathogenic microbes which occur in nature and the gastrointestinal tract of ruminant animals, where they pose a positive effect on the physiology of the host (Dunne et al., 1999). Probiotics improve microbial ecosystem (Sandine, 1979; Musa et al., 2009), nutrient synthesis and their bio-availability resulting in better growth performance in farm animals (Oyetayo and Oyetayo, 2005). In addition to these probiotics also improve nutrient absorption (Teeler and Vanabelle, 1991), reduce the incidence of intestinal infection (Casas and Dobrogosz, 2000) and restore the gut micro flora in case of diarrhoea 
(Musa et al., 2009). Known to increase ruminal $\mathrm{pH}$ (Umberger and Notter, 1989), total volatile fatty acids (VFAs) and ruminal biomass (Newbolt et al., 1996) and thus influence the cellulolytic activity and microbial protein synthesis and fiber degradation (Martin and Nisbet, 1990; Yoon and Stern, 1996). It is also considered that they compete with other pathogenic microorganisms for the provision of nutrients and other growth factors (Rolfe, 2000). They enhance immunity (Aattouri et al., 2001) by promoting the antibodies, $\operatorname{IgA}$ and cytokines production (Trebichavsky and Splichal, 2006). A positive impact of probiotics supplementation on nutrient intake, weight gain and feed conversion ratio (FCR) in ruminants has been reported by many workers (Chiofalo et al., 2004; Antunovic et al., 2006; Whitley et al., 2009). It is further stated that probiotics can stimulate specific groups of beneficial bacteria in the rumen, and has provided mechanistic models that can explain their effects on animal performance (Dutta et al., 2009).

\section{Materials and Methods}

Sirohi and Barbari kids just after weaning at the age of 2-3 month of either sex were selected and distributed randomly in different groups. i.e. Twenty four (24) Sirohi and Twenty four (24) Barbari male and female kids were randomly selected from the Institute's flock for the study. Every care was taken while selecting the animals, so that they had equal body weight and almost same age. These animals were randomly distributed into four different groups (6 kids in each group), i.e., Treatment - 1 (Control) $\left(\mathrm{T}_{1}\right)$, Treatment-2 $\left(\mathrm{T}_{2}\right)$, Treatment-3 $\left(\mathrm{T}_{3}\right)$ and Treatment-4 $\left(\mathrm{T}_{4}\right)$.

\section{Body weight (BW) at monthly interval}

The body weight of Barbari and Sirohi kids were recorded at the onset of the experiment and then at monthly interval till the end of the experiment and are presented in Table 1 and figure 1. It was calculated by dividing body weight by total number of days.

Table revealed that average body weight $(\mathrm{kg})$ of Barbari kids at the start of experiment were $8.58 \pm 0.34,8.51 \pm 0.34,8.66 \pm 0.29,8.54 \pm$ 0.21 and Sirohi kids were $9.41 \pm 0.34,9.34 \pm$ $0.34,9.43 \pm 0.29,9.37 \pm 0.21$ in $\mathrm{T}_{1}, \mathrm{~T}_{2}, \mathrm{~T}_{3}$ and $\mathrm{T}_{4}$ groups respectively.

Average body weight $(\mathrm{kg})$ of Barbari kidsat the end of experiment were $14.38 \pm 0.24,15.25$ $\pm 0.23,15.81 \pm 0.26,16.15 \pm 0.19$ and Sirohi kids were15.00 $\pm 0.24,15.87 \pm 0.23,16.42 \pm$ $0.26,16.77 \pm 0.91$ in $\mathrm{Ti}, \mathrm{T}_{2}, \mathrm{~T}_{3}$ and $\mathrm{T}_{4}$ group respectively.

Statistical analysis revealed that the mean body weight of $\mathrm{T}_{3}$ and $\mathrm{T}_{4}$ group was differed significantly $(\mathrm{P}<0.05)$ from $\mathrm{T}_{1}$ group and no significant difference was observed between $\mathrm{T}_{1}$ and $\mathrm{T}_{2}$ group of Barbari and Sirohi kids. Highest value of body weight was observed in $\mathrm{T}_{4}$ group and lowest value was observed in $\mathrm{T}_{1}$ group at monthly interval in both the breeds.

\section{Average monthly body weight gain}

The average monthly body weight gain (kg/animal) of Barbari and Sirohi kids in different groups are presented in Table 2 and figure 2. Total weight gain (TWG, $\mathrm{kg}$ ) was calculated as the difference between final and initial weights. Growth performance indices were calculated as follows: Growth rate $(\%)=$ (Final weight - initial weight) / (initial weight)*100

The mean body weight gain ( $\mathrm{kg} / \mathrm{animal})$ of Barbari kids after one month of experiment was $0.38 \pm 0.01,0.36 \pm 0.08,0.44 \pm 0.01,0.44$ \pm 0.01 andin Sirohi kids were $0.37 \pm 0.02,0.36$ \pm 0.08 and $0.44 \pm 0.01,0.44 \pm 0.01$ for $\mathrm{T}_{1}, \mathrm{~T}_{2}, \mathrm{~T}_{3}$ and $\mathrm{T}_{4}$ group respectively. 


\section{Body length}

The average body length $(\mathrm{cm})$ of Barbari and Sirohi kids in different groups are presented in Table 3 and figure 3 . The average body length $(\mathrm{cm})$ at the start of the experiment was observed as $36.49 \pm 0.47,37.26 \pm 0.54,37.36 \pm$ 0.51 and $37.54 \pm 0.56$ in Barbari kids and $37.80 \pm 0.47,38.57 \pm 0.54,38.67 \pm 0.51$ and $38.85 \pm 0.56$ in Sirohi kids for $\mathrm{T}_{1}, \mathrm{~T}_{2}, \mathrm{~T}_{3}$ and $\mathrm{T}_{4}$ group respectively.

At the end of the experiment average body length $(\mathrm{cm})$ in Barbari kids were recorded as $57.58 \pm 0.25,60.20 \pm 0.53,60.26 \pm 0.31$ and $59.40 \pm 0.59$ and in Sirohi kids were recorded as $59.04 \pm 0.25,61.66 \pm 0.53,61.66 \pm 0.53$ and $60.86 \pm 0.59$ for $\mathrm{T}_{1}, \quad \mathrm{~T}_{2}, \mathrm{~T}_{3}$ and $\mathrm{T}_{4}$ group respectively.

Statistical analysis revealed no significant difference in average body length of Barbari and Sirohi kids between different treatment groups.

\section{Results and Discussion}

\section{Body weight (BW) at monthly interval}

Average body weight $(\mathrm{kg})$ of Barbari kidsat the end of experiment were $14.38 \pm 0.24,15.25$ $\pm 0.23,15.81 \pm 0.26,16.15 \pm 0.19$ and Sirohi kids were15.00 $\pm 0.24,15.87 \quad \pm 0.23,16.42 \quad \pm$ $0.26,16.77 \pm 0.91$ in $\mathrm{Ti}, \mathrm{T}_{2}, \mathrm{~T}_{3}$ and $\mathrm{T}_{4}$ group respectively.

Statistical analysis revealed that the mean body weight of $\mathrm{T}_{3}$ and $\mathrm{T}_{4}$ group was differed significantly $(\mathrm{P}<0.05)$ from $\mathrm{T}_{1}$ group and no significant difference was observed between $\mathrm{T}_{1}$ and $\mathrm{T}_{2}$ group of Barbari and Sirohi kids. Highest value of body weight was observed in $\mathrm{T}_{4}$ group and lowest value was observed in $\mathrm{T}_{1}$ group at monthly interval in both the breeds

\section{Average monthly body weight gain}

The overall body weight gain $(\mathrm{kg})$ during experimental period of Barbari kids was 0.55 $\pm 0.01,0.72 \pm 0.03,0.76 \pm 0.03$ and $0.82 \pm 0.03 \mathrm{~kg}$ and of Sirohi kids was $0.55 \pm 0.01,0.55 \pm 0.02$, $0.76 \pm 0.03$ and $0.82 \pm 0.03$ for $\mathrm{T}_{1}, \mathrm{~T}_{2}, \mathrm{~T}_{3}$ and $\mathrm{T}_{4}$ group respectively.

Statistical analysis revealed that the mean body weight gain of $T_{2}$ and $T_{4}$ group in Barbari and Sirohi kids was differed significantly $(\mathrm{P}<0.05)$ from $\mathrm{T}_{1}$ and non significant difference was observed between $\mathrm{T}_{2}$ and $\mathrm{T}_{3}$ groups. Highest value of body weight gain was observed in $\mathrm{T}_{4}$ group and lowest value was observed in $T_{1}$ group at monthly interval in both the breeds (Table 4).

Table.1 Animal distribution and Diet

\begin{tabular}{|c|c|c|}
\hline Groups & No. of animals & Feeding with 5-6 hours grazing \\
\hline $\begin{array}{c}\text { (Control) } \\
\mathrm{T}_{1}\end{array}$ & $\begin{array}{l}6 \text { barbari }+6 \text { sirohi kidsof } 3-4 \\
\text { months of age }\end{array}$ & $\begin{array}{l}\text { Grazing+ concentrate feeding } \\
\text { (@100 g/day/kid) }\end{array}$ \\
\hline $\mathrm{T}_{2}$ & $\begin{array}{l}6 \text { barbari }+6 \text { sirohiof3-4 } \\
\text { months of age }\end{array}$ & $\mathrm{T}_{1}+$ S.cerevisiae (@2g/animal /day) \\
\hline $\mathrm{T}_{3}$ & $\begin{array}{l}6 \text { barbari }+6 \text { sirohi kids of } 3-4 \\
\text { months of age }\end{array}$ & $\mathrm{T}_{1}+@$ @50gextra concentrate feeding \\
\hline $\mathrm{T}_{4}$ & $\begin{array}{l}6 \text { barbari }+6 \text { sirohiof } 3-4 \\
\text { months of age }\end{array}$ & $\begin{array}{l}\mathrm{T}_{1}+50 \mathrm{~g} \text { extra concentrate feeding }+S \\
\text { cerevisiae(@2g/animal /day). }\end{array}$ \\
\hline
\end{tabular}


Table.2 Average monthly Body weight $(\mathrm{kg})$ of Barbari and Sirohi Goat

\begin{tabular}{|c|c|c|c|c|c|c|c|c|}
\hline \multirow{2}{*}{$\begin{array}{c}\text { Mont } \\
\text { hs/ } \\
\text { Bree } \\
\text { ds }\end{array}$} & \multicolumn{2}{|c|}{$T_{1}$} & \multicolumn{2}{|c|}{$\mathbf{T}_{2}$} & \multicolumn{2}{|c|}{$\mathbf{T}_{3}$} & \multicolumn{2}{|r|}{$\mathbf{T}_{4}$} \\
\hline & Barbari & Sirohi & Barbari & Sirohi & Barbari & Sirohi & Barbari & Sirohi \\
\hline Initia & $\begin{array}{l}8.58^{\mathrm{a}} \\
\pm 0.34\end{array}$ & $\begin{array}{c}9.41^{\mathrm{a}} \pm \\
0.34\end{array}$ & $\begin{array}{c}8.51^{\mathrm{ab}} \pm \\
0.34\end{array}$ & $\begin{array}{c}9.34^{\mathrm{ab}} \pm \\
0.34\end{array}$ & $\begin{array}{c}8.66^{\mathrm{b}} \pm \\
0.29\end{array}$ & $\begin{array}{c}9.43^{\mathrm{b}} \pm \\
0.29\end{array}$ & $\begin{array}{c}8.54^{\mathrm{b}} \pm \\
0.21\end{array}$ & $9.37^{b} \pm 0.21$ \\
\hline 1 & $\begin{array}{l}9.34^{\mathrm{a}} \\
\pm 0.23\end{array}$ & $\begin{array}{l}9.96^{\mathrm{a}} \\
\pm 0.23\end{array}$ & $\begin{array}{l}9.55^{\mathrm{ab}} \\
\pm 0.22\end{array}$ & $\begin{array}{c}10.17^{\mathrm{ab}} \\
0.22\end{array}$ & $\begin{array}{l}9.62^{b} \\
\pm 0.22\end{array}$ & $\begin{array}{c}10.24^{\mathrm{b}} \pm \\
0.22\end{array}$ & $\begin{array}{l}9.56^{\mathrm{b}} \\
\pm 0.17\end{array}$ & $10.18^{b} \pm 0.17$ \\
\hline 2 & $\begin{array}{l}10.13^{\mathrm{a}} \\
\pm 0.23\end{array}$ & $\begin{array}{l}10.75^{\mathrm{a}} \\
\pm 0.23\end{array}$ & $\begin{array}{c}10.33^{\mathrm{ab}} \\
\pm 0.21\end{array}$ & $\begin{array}{c}10.95^{\mathrm{ab}} \\
\pm 0.21\end{array}$ & $\begin{array}{l}10.63^{\mathrm{b}} \\
\pm 0.22\end{array}$ & $\begin{array}{l}11.25^{b} \\
\pm 0.22\end{array}$ & $\begin{array}{l}10.62^{\mathrm{b}} \\
\pm 0.16\end{array}$ & $11.24^{b} \pm 0.16$ \\
\hline 3 & $\begin{array}{c}10.90^{\mathrm{a}} \pm 0 \\
23\end{array}$ & $\begin{array}{c}11.52^{\mathrm{a}} \pm \\
0.23\end{array}$ & $\begin{array}{c}11.27^{\mathrm{ab}} \pm 0 \\
21\end{array}$ & $\begin{array}{c}11.89^{\mathrm{ab}} \\
\pm 0.21\end{array}$ & $\begin{array}{l}11.63^{b} \\
\pm 0.21\end{array}$ & $\begin{array}{c}12.25^{\mathrm{b}} \pm \\
0.21\end{array}$ & $\begin{array}{l}11.63^{b} \\
\pm 0.15\end{array}$ & $12.25^{\mathrm{b}} \pm 0.15$ \\
\hline 4 & $\begin{array}{c}11.73 \\
\pm 0.24^{\mathrm{a}}\end{array}$ & $\begin{array}{l}12.35^{\mathrm{a}} \\
\pm 0.24\end{array}$ & $\begin{array}{c}12.16^{\mathrm{ab}} \\
\pm 0.21\end{array}$ & $\begin{array}{c}12.76^{\mathrm{ab}} \\
\pm 0.21\end{array}$ & $\begin{array}{l}12.53^{b} \\
\pm 0.22\end{array}$ & $\begin{array}{l}13.15^{\mathrm{b}} \\
\pm 0.22\end{array}$ & $\begin{array}{l}12.59^{b} \\
\pm 0.16\end{array}$ & $13.21^{\mathrm{b}} \pm 0.16$ \\
\hline 5 & $\begin{array}{l}12.51^{\mathrm{a}} \\
\pm 0.24\end{array}$ & $\begin{array}{l}13.15^{\mathrm{a}} \\
\pm 0.24\end{array}$ & $\begin{array}{c}13.00^{\mathrm{ab}} \\
\pm 0.21\end{array}$ & $\begin{array}{c}13.62^{\mathrm{ab}} \\
\pm 0.21\end{array}$ & $\begin{array}{l}13.40^{b} \\
\pm 0.23\end{array}$ & $\begin{array}{l}14.02^{b} \\
\pm 0.23\end{array}$ & $\begin{array}{l}13.52^{b} \\
\pm 0.15\end{array}$ & $14.14^{\mathrm{b}} \pm 0.15$ \\
\hline 6 & $\begin{array}{l}13.32^{\mathrm{a}} \\
\pm 0.24\end{array}$ & $\begin{array}{l}13.94^{\mathrm{a}} \\
\pm 0.24\end{array}$ & $\begin{array}{c}13.96^{\mathrm{ab}} \\
\pm 0.21\end{array}$ & $\begin{array}{c}14.58^{\mathrm{ab}} \\
\pm 0.21\end{array}$ & $\begin{array}{l}14.42^{b} \\
\pm 0.24\end{array}$ & $\begin{array}{c}15.04^{\mathrm{b}} \pm \\
0.24\end{array}$ & $\begin{array}{l}14.64^{b} \\
\pm 0.17\end{array}$ & $15.26^{\mathrm{b}} \pm 0.71$ \\
\hline 7 & $\begin{array}{l}14.38^{\mathrm{a}} \\
\pm 0.24\end{array}$ & $\begin{array}{l}15.00^{\mathrm{a}} \\
\pm 0.24\end{array}$ & $\begin{array}{c}15.25^{\mathrm{ab}} \pm 0 \\
23\end{array}$ & $\begin{array}{c}15.87^{\mathrm{ab}} \\
\pm 0.23\end{array}$ & $\begin{array}{l}15.81^{\mathrm{b}} \\
\pm 0.26\end{array}$ & $\begin{array}{c}16.42^{\mathrm{b}} \pm \\
0.26\end{array}$ & $\begin{array}{l}16.15^{b} \\
\pm 0.19\end{array}$ & $16.77^{b} \pm 0.91$ \\
\hline
\end{tabular}

Table.3 Average monthly body weight gain (kg/animal) of Barbari and Sirohi goat

\begin{tabular}{|c|c|c|c|c|c|c|c|c|}
\hline \multirow{2}{*}{$\begin{array}{l}\text { Month } \\
\text { s/ } \\
\text { Breeds }\end{array}$} & \multicolumn{2}{|c|}{$T_{1}$} & \multicolumn{2}{|c|}{$T_{2}$} & \multicolumn{2}{|c|}{$T_{3}$} & \multicolumn{2}{|c|}{$\mathrm{T}_{4}$} \\
\hline & Barbari & Sirohi & Barbari & Sirohi & Barbari & Sirohi & Barbari & Sirohi \\
\hline Initial & - & - & - & - & 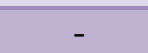 & - & - & 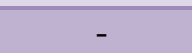 \\
\hline 1 & $0.38^{\mathrm{a}} \pm 0.01$ & $0.37^{\mathrm{a}} \pm 0.02$ & $0.36^{b} \pm 0.08$ & $\begin{array}{l}0.36^{\mathrm{b}} \\
\pm 0.08\end{array}$ & $\begin{array}{l}0.44^{\mathrm{bc}} \\
\pm 0.01\end{array}$ & $\begin{array}{l}0.44^{\mathrm{bc}} \\
\pm 0.01\end{array}$ & $\begin{array}{l}0.44^{\mathrm{c}} \\
\pm 0.01\end{array}$ & $0.44^{\mathrm{c}} \pm 0.01$ \\
\hline 2 & $0.39^{\mathrm{a}} \pm 0.02$ & $0.38^{\mathrm{a}} \pm 0.01$ & $0.42^{\mathrm{b}} \pm 0.01$ & $\begin{array}{l}0.42^{\mathrm{b}} \\
\pm 0.01\end{array}$ & $\begin{array}{l}0.44^{\mathrm{bc}} \\
\pm 0.01\end{array}$ & $\begin{array}{l}0.44^{\mathrm{bc}} \\
\pm 0.01\end{array}$ & $\begin{array}{l}0.48^{\mathrm{c}} \\
\pm 0.02\end{array}$ & $\begin{array}{c}0.48^{\mathrm{c}} \pm \\
0.02\end{array}$ \\
\hline 3 & $0.40^{\mathrm{a}} \pm 0.01$ & $0.39^{\mathrm{a}} \pm 0.02$ & $0.43^{b} \pm 0.01$ & $\begin{array}{l}0.43^{\mathrm{b}} \\
\pm 0.01\end{array}$ & $\begin{array}{l}0.49^{\mathrm{bc}} \\
\pm 0.02\end{array}$ & $\begin{array}{l}0.49^{\mathrm{bc}} \\
\pm 0.02\end{array}$ & $\begin{array}{l}0.50^{\mathrm{c}} \\
\pm 0.17\end{array}$ & $\begin{array}{c}0.50^{c} \pm \\
0.17\end{array}$ \\
\hline 4 & $0.41^{\mathrm{a}} \pm 0.01$ & $0.40^{\mathrm{a}} \pm 0.01$ & $0.48^{\mathrm{b}} \pm 0.01$ & $\begin{array}{l}0.43^{\mathrm{b}} \\
\pm 0.02\end{array}$ & $\begin{array}{l}0.51^{\mathrm{bc}} \\
\pm 0.01\end{array}$ & $\begin{array}{l}0.51^{\mathrm{bc}} \\
\pm 0.01\end{array}$ & $\begin{array}{l}0.53^{\mathrm{c}} \\
\pm 0.01\end{array}$ & $\begin{array}{c}0.53^{\mathrm{c}} \pm \\
0.01\end{array}$ \\
\hline 5 & $0.43^{\mathrm{a}} \pm 0.02$ & $0.41^{\mathrm{a}} \pm 0.01$ & $0.51^{b} \pm 0.02$ & $\begin{array}{l}0.48^{\mathrm{b}} \\
\pm 0.01\end{array}$ & $\begin{array}{l}0.54^{\mathrm{bc}} \\
\pm 0.02\end{array}$ & $\begin{array}{l}0.53^{\mathrm{bc}} \\
\pm 0.01\end{array}$ & $\begin{array}{l}0.62^{c} \\
\pm 0.02\end{array}$ & $\begin{array}{c}0.54^{c} \pm \\
0.01\end{array}$ \\
\hline 6 & $\begin{array}{c}0.47^{\mathrm{a}} \pm \\
0.04\end{array}$ & $0.43^{\mathrm{a}} \pm 0.02$ & $0.66^{\mathrm{b}} \pm 0.04$ & $\begin{array}{c}0.55^{b} \pm 0.0 \\
1\end{array}$ & $\begin{array}{c}0 . .63^{\mathrm{bc}} \pm \\
0.04\end{array}$ & $\begin{array}{l}0.54^{\mathrm{bc}} \\
\pm 0.02\end{array}$ & $\begin{array}{c}0.65^{\mathrm{c}} \pm \\
0.03\end{array}$ & $0.62^{\mathrm{c}} \pm 0.02$ \\
\hline 7 & $0.55^{\mathrm{a}} \pm 0.01$ & $0.55^{\mathrm{a}} \pm 0.01$ & $0.72^{\mathrm{b}} \pm 0.03$ & $\begin{array}{c}0.55^{\mathrm{b}} \pm \\
0.02\end{array}$ & $\begin{array}{l}0.76^{\mathrm{bc}} \\
\pm 0.03\end{array}$ & $\begin{array}{l}0.76^{\mathrm{bc}} \\
\pm 0.03\end{array}$ & $\begin{array}{l}0.82^{\mathrm{c}} \\
\pm 0.03\end{array}$ & $0.82^{\mathrm{c}} \pm 0.03$ \\
\hline
\end{tabular}

* Group I ( $\left.\mathrm{T}_{1}\right)$-Grazing + concentrate feeding (@100 g / day / kid), ** $\mathrm{T}_{1}+$ S. cerevisiae (@ 2g / animal / day), *** $\mathrm{T}_{1}+$ @ 50g extra concentrate feeding, $* * * * \mathrm{~T}_{1}+50 \mathrm{~g}$ extra concentrate feeding + S. cerevisiae (@ $2 \mathrm{~g} /$ animal /day). The values with different superscripts within a row (a, b and c) differ significantly $(\mathrm{P}<0.05)$. 
Table.4 Average monthly body length $(\mathrm{cm})$ of Barbari and Sirohi Kids

\begin{tabular}{|c|c|c|c|c|c|c|c|c|}
\hline \multirow{2}{*}{$\begin{array}{c}\text { Months/ } \\
\text { Breeds }\end{array}$} & \multicolumn{2}{|c|}{$\mathbf{T}_{\mathbf{1}}$} & \multicolumn{2}{c|}{$\mathbf{T}_{\mathbf{2}}$} & \multicolumn{2}{c|}{$\mathbf{T}_{\mathbf{3}}$} & \multicolumn{2}{c|}{$\mathbf{T}_{\mathbf{4}}$} \\
\hline Initial & Barbari & Sirohi & Barbari & Sirohi & Barbari & Sirohi & Barbari & Sirohi \\
& $36.49 \pm$ & $37.80 \pm$ & $37.26 \pm$ & $38.57 \pm 0.54$ & $37.36 \pm$ & $38.67 \pm$ & $37.54 \pm 0.56$ & 38.85 \\
\hline 1 & 0.47 & 0.47 & 0.54 & & 0.51 & 0.51 & & \pm 0.56 \\
\hline & 40.08 & 41.38 & 41.00 & $42.30 \pm 0.95$ & $41.63 \pm$ & 42.93 & $41.50 \pm 0.50$ & 42.80 \\
& \pm 0.48 & \pm 0.48 & \pm 0.95 & & 0.81 & \pm 0.81 & & \pm 0.50 \\
\hline 2 & 43.67 & 45.00 & 44.79 & $46.12 \pm 0.89$ & 45.79 & 47.12 & $45.15 \pm 0.63$ & 46.48 \\
& \pm 0.46 & \pm 0.46 & \pm 0.89 & & \pm 0.70 & \pm 0.70 & & \pm 0.63 \\
\hline 3 & 46.96 & 48.25 & 48.43 & $49.72 \pm 0.79$ & 49.59 & 50.88 & $48.76 \pm 0.80$ & 50.05 \\
& \pm 0.32 & \pm 0.32 & \pm 0.79 & & \pm 0.55 & \pm 0.55 & & \pm 0.80 \\
\hline 4 & 50.13 & 51.43 & 52.00 & $53.30 \pm 0.87$ & 52.97 & 54.27 & $52.22 \pm 1.06$ & 53.52 \\
& \pm 0.13 & \pm 0.13 & \pm 0.87 & & \pm 0.63 & \pm 0.63 & & \pm 1.06 \\
\hline 5 & 53.33 & 54.67 & 55.61 & $56.95 \pm 0.78$ & 56.48 & 57.82 & $55.59 \pm 0.78$ & 56.93 \\
& \pm 0.19 & \pm 0.19 & \pm 0.78 & & \pm 0.57 & \pm 0.57 & & \pm 0.78 \\
\hline 6 & 56.62 & 57.90 & 59.24 & $60.52 \pm 0.53$ & 59.30 & 60.52 & $58.44 \pm 0.59$ & 59.72 \\
& \pm 0.25 & \pm 0.25 & \pm 0.53 & & \pm 0.31 & \pm 0.58 & & \pm 0.59 \\
\hline 7 & 57.58 & 59.04 & 60.20 & $61.66 \pm 0.53$ & 60.26 & 61.66 & $59.40 \pm 0.59$ & 60.86 \\
& \pm 0.25 & \pm 0.25 & \pm 0.53 & & \pm 0.31 & \pm 0.53 & & \\
\hline
\end{tabular}

Fig.1 Average monthly Body weight (kg) of Barbari and Sirohi Goat

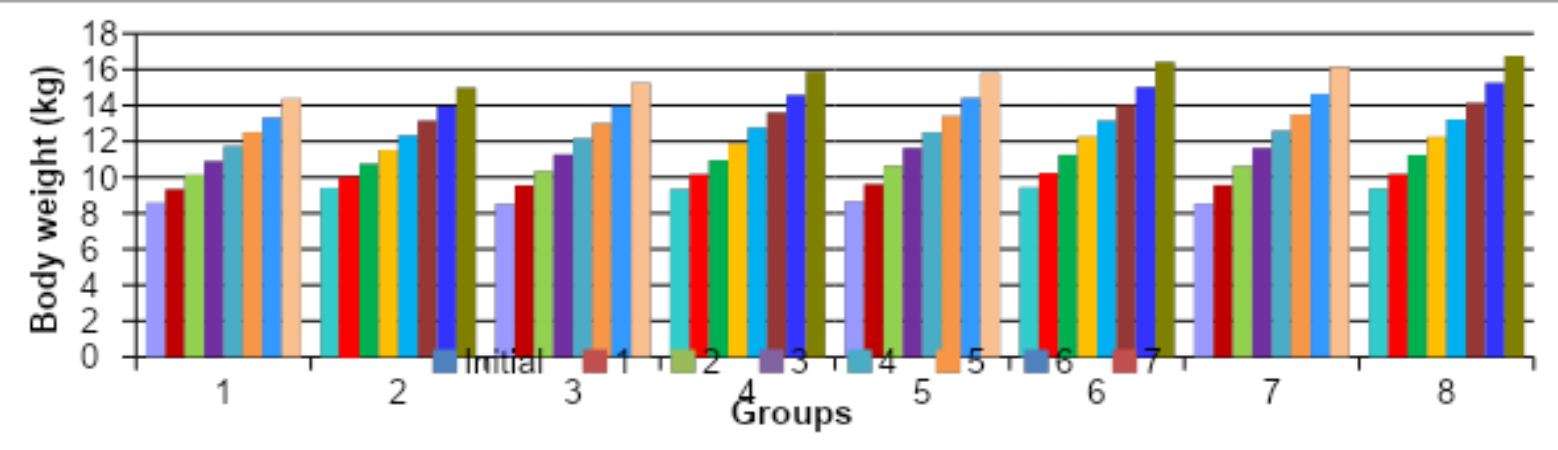

Fig.2 Average monthly body weight gain (kg/animal) of Barbari and Sirohi goat

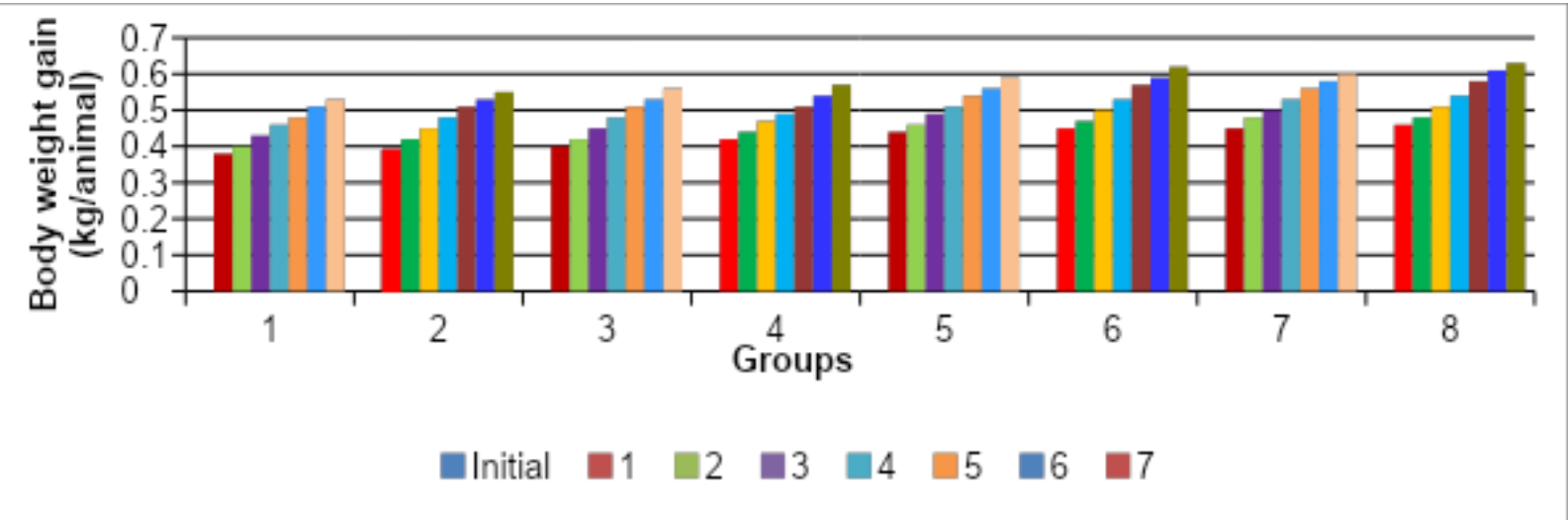


Fig.3 Average monthly body length $(\mathrm{cm})$ of Barbari and Sirohi Kids

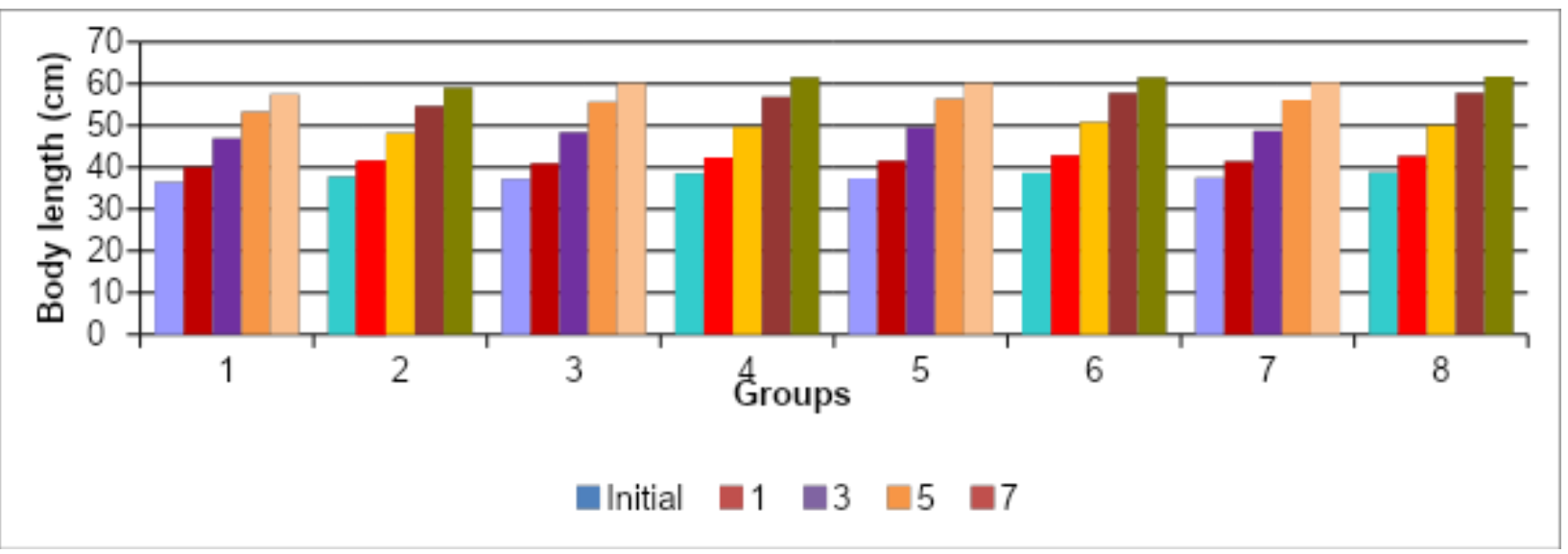

\section{Average monthly body length $(\mathrm{cm})$}

At the end of the experiment average body length $(\mathrm{cm})$ in Barbari kids were recorded as $57.58 \pm 0.25,60.20 \pm 0.53,60.26 \pm 0.31$ and $59.40 \pm 0.59$ and in Sirohi kids were recorded as $59.04 \pm 0.25,61.66 \pm 0.53,61.66 \pm 0.53$ and $60.86 \pm 0.59$ for $\mathrm{T}_{1}, \mathrm{~T}_{2}, \mathrm{~T}_{3}$ and $\mathrm{T}_{4}$ group respectively.

Statistical analysis revealed no significant difference in average body length of Barbari and Sirohi kids between different treatment groups.

\section{References}

Aattouri, N., Bouras, M., Tome, D., Marcos, A. and Lemonnier, D. (2001). Oral ingestion of lactic acid bacteria by rats increases lymphocyte proliferation and interferon production. British Journal of Nutrition, 87: 367-373.

Antunovic, Z., Speranda, M., Amidzic, D., Seric, V and Stainer, Z. (2006). Probiotic applications in lambs nutrition. Krmiva48: 175-180.

Casas and Dobrogosz, (2000) Validation of the Probiotic Concept: Lactobacillus reuteri Confers Broad-spectrum
Protection against Disease in Humans and Animals Microbial Ecology in Health and Disease Volume 12: 247285

Chiofalo, V., Liotta, L. and Chiofalo, B. (2004). Effects of the administration of lactobacilli on body growth and on the metabolic profile in growing Maltese goat kids. Reprod. Nutr. Dev., 44: 449457.

Dunne, C. O., Mahony, L., Murphy, L.O., Halloran, S., Feeney, M., Flynn, S., Fitzgerald, G., Daly, C., Kiely, B.O., Sullivan, G., Shanahan, F. and Collins, J.K. (1999). Probiotics; from myth to reality-Demonstration of functionality in animal models of disease and in human clinical trials. Antonie Van Leeuwenhoek, 76: 279-292.

Dutta, T.K., kundu, s.s. and kumar, M.(2009). potential of direct fed micro organism on lactation performance in ruminant a critical review. Livestock research for rural development, 21(160).

Mousa, Kh., El-Malky, M., Komonna, OM, O.F. and Rashwan, S. E. (2009). Effect of some yeast and minerals on the productive and reproductive performance. Ruminants Journal of American Science, 8(2): 212-214. 
Musa et al., Mousa, Kh., El-Malky, M., Komonna, OM, O.F. and Rashwan, S. E (2009). Effect of some yeast and minerals on the productive and reproductive performance. Ruminants Journal of American Science, 8(2): 212-214.

Newbold, CJ., Wallace RJ, FM Mcintosh, (1996). Mode of action of the yeast saccharomyces cerevisiae as a feed additive for ruminants British journal of nutrition, 76, (2): 249-261.

Nisbet, DJ., and Martin SA (1990). Effect of dicarboxylic acids and Aspergillus oryzae fermentation extract on lactate uptake by the ruminal bacterium Selenomonas ruminantium. Appl. Environ. Microbiol., 68(7): 2142-9 .

Oyetayo and Oyetayo, (2005). Potential of probiotics as biotherapeutic agents targeting the innate immune system African Journal of Biotechnology. 4(2): 123-127.

Rolfe, R.D (2000) The role of probiotic cultures in the control of gastrointestinal health. Journal of nutrition. 130(2s suppl): 396s-402s

Teeler and Vanabelle, (1991), Use of probiotics in gastrointestinal disorders: what to recommend Therap Advance Gastroenterol. 3(5): 307-319

Trebichavsky and Splichal, (2006). Nutritional studies on green forage. Ph.D. Thesis. (Animal Nutrition), Ain shams University, Cairo, Egypt

Umberger, S.H.; McClure, W.H. -White, H.E.Notter, O.R. Blacksburg, VA: The Station. Animal science research report - Virginia Polytechnic Institute and State African Journal of Agricultural Research. 6(23). 5198-5203,

Whitley, N.C., Cazac, D., Rude, B.J., JacksonO'Brien, D. and Parveen, S. (2009). Use of commercial Probiotics supplement in meat goat. Journal of Animal Science, 87: 723-728.

Yoon and Stern, (1996). Effects of Saccharomyces cerevisiae and Aspergillus oryzae cultures on ruminal fermentation in dairy cows. Journal of Dairy Science.79(3): 411-7

\section{How to cite this article:}

Gautam, V. N., Shraddha Shrivastava, G. P. Lakhani, R. P. S. Baghel, B. Roy, A. Jain and Rahul Shehar. 2019. Effect of Probiotics (Saccharomyces cerevisiae) Supplementation on Growth Performance of Growing Barbari and Sirohi Goats. Int.J.Curr.Microbiol.App.Sci. 8(12): 225-231. doi: https://doi.org/10.20546/ijcmas.2019.812.032 